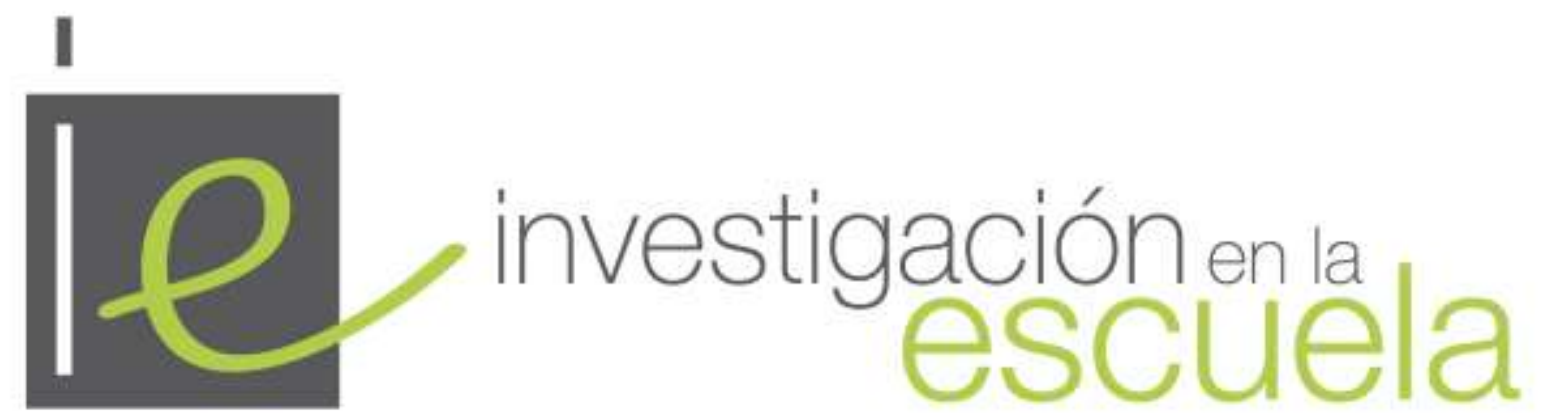

Revista internacional de investigación e innovación educativa

\title{
La internacionalización de la Educación Infantil a través de un proyecto $e T$ winning entre España y Francia
}

\author{
Belén Zamanillo Mateo ${ }^{1}$, Esther Sanz de la Cal' y Josefa Ramos Rodríguez ${ }^{2}$ \\ Universidad de Burgos ${ }^{1}$ y CEIP Fernando de Rojas ${ }^{2}$ \\ España
}

Citación: Zamanillo-Mateo, B., Sanz de la Cal, E. y Ramos-Rodríguez, J. (2018). La internacionalización de la Educación Infantil a través de un proyecto éTwinning entre España y Francia. Investigación en la Escuela, 96, 33-49.

Resumen: Este artículo se centra en el desarrollo del enfoque intercultural en la enseñanza de lenguas extranjeras en Educación Infantil a través de la movilidad virtual. En este estudio se presenta el desarrollo de la puesta en práctica de un proyecto eTwinning realizado en el área de Lengua Extranjera (inglés) en una clase de tercero de Educación Infantil con la colaboración de un colegio francés. Además, se estableció la valoración del impacto de dicho proyecto en los alumnos participantes junto a sus profesoras de los respectivos países. Los resultados ofrecen una visión muy positiva del impacto de este tipo de proyectos en los alumnos, profesores y centros educativos participantes. Por lo tanto, este proyecto eTwinning se ha implementado de manera muy satisfactoria, aumentando así la motivación de los alumnos hacia el aprendizaje del inglés desde una perspectiva intercultural.

Palabras clave: "Interculturalidad"; "Educación Infantil"; "movilidad virtual"; "eTwinning".

The internationalization of Early Childhood Education through an $e T$ winning project between Spain and France

Abstract: This paper is focused on the intercultural approach in the teaching of foreign languages in Pre-Primary Education by virtual mobility. This essay presents the implementation of an eTwinning 
project carried out in the Foreign Language area (English) in a 3rd Pre-Primary class in collaboration with a French school. Besides, the assessment of the impact of the project in the involved students with their teachers of each country is established. The results offer a positive view of the impact of this kind of projects in the students, teachers and schools involved. Therefore, this eTwinning proyect has been carried out satisfactorily improving the students' motivation.

Key words: "Interculturality"; "Pre-Primary Education"; "virtual mobility"; "eTwinning".

\section{L'internationalisation de l'éducation de la petite enfance à travers un projet $e T_{w i n n i n g}$ entre l'Espagne et la France}

Resumè: Cet article recentre le développement de l'approche interculturelle dans l'enseignement des langues étrangères dans l'école maternelle à travers de la mobilité virtuelle. Cette étude présente le développement de la mise en œuvre d'un projet eTwinning mené dans le domaine des langues étrangères (anglais) dans une classe de Grande section à l'école maternelle avec la collaboration d'une école française. En outre, l'évaluation de l'impact de ce projet sur les étudiants participants a été établie avec leurs enseignants des pays respectifs. Les résultats offrent une vision très positive de l'impact de ce type de projet sur les élèves, les enseignants et les écoles participantes. Par conséquent, ce projet eTwinning a été mis en œuvre de manière très satisfaisante, augmentant ainsi la motivation des étudiants pour l'apprentissage de l'anglais dans une perspective interculturelle. Mots-clés: "Interculturalité"; "Éducation de la petite enfance"; "mobilité virtuelle"; "eTwinning".

\section{Introducción}

El artículo versa sobre la importancia de las perspectivas interculturales y la movilidad virtual en el marco del aprendizaje del inglés como lengua extranjera. El objetivo principal es ofrecer una propuesta de un proyecto eTwinning para el tercer curso de Educación Infantil, que desarrolle la competencia comunicativa intercultural entre los alumnos, y analizar la valoración del proyecto por parte de los participantes al igual que investigar si se produce un aumento de la motivación hacia las lenguas y sus culturas a lo largo del mismo. Este proyecto de hermanamiento eTwinning se llevó a cabo entre dos colegios de España y Francia durante dos meses en el curso 2017-2018.

$\mathrm{El}$ aprendizaje y enseñanza de idiomas debe fomentar un mayor entendimiento de la sociedad y cultura de otros hablantes. Enseñar una lengua es enseñar su cultura, la competencia intercultural y la competencia lingüística están íntimamente relacionadas. En nuestra sociedad del siglo XXI, las Tecnologías de la Información y la Comunicación (TIC) han tenido un gran impacto social al romper las barreras que limitan el acceso y el intercambio de información, de tal forma que han permitido abrir las puertas de las aulas y que los alumnos viajen y conozcan aspectos culturales de otros lugares. Gracias a las TIC, se puede despertar el interés de los niños por otras culturas y el sentimiento de ser ciudadanos de un mundo que considera la diversidad cultural como un enriquecimiento personal y social.

La importancia de iniciativas como esta, que impliquen un contacto directo con hablantes de otras lenguas y con otras culturas, ha sido resaltada recientemente por el European Council (2017) a través de sus políticas lingüísticas que intentan proteger la diversidad lingüística en la Unión Europea. Según la UNESCO (2006), la diversidad cultural permite comprender la cultura propia y reconocer la existencia de muchas otras en un plano de igualdad. De la misma manera, existen numerosos ejemplos de la literatura de la importancia de la movilidad virtual en el desarrollo de la competencia comunicativa intercultural (Byram, 2008; Byram, 2014, Byram y Fleming, 2001; Council of Europe, 2015; O’Dowd, 2015; Sevilla-Pavón y Haba-Osca, 2016; Valls, 2011). Por lo tanto, educar en la interculturalidad ha de estar presente en el aula de lengua extranjera. En palabras de 
Leiva (2010), con la intención de que los centros escolares se abran a diferentes maneras de ver, sentir y comprender la realidad social y educativa, es necesario implicar a toda la comunidad educativa desde maestros, familias y alumnos.

\section{Marco teórico}

\section{La competencia comunicativa intercultural en el aprendizaje de idiomas}

$\mathrm{El}$ aprendizaje de una lengua extranjera conlleva cierto grado de aprendizaje intercultural. $\mathrm{Al}$ mismo tiempo que se aprende un idioma y una cultura diferente, se es más consciente de la propia. En ese contacto entre diversas lenguas y culturas reside la competencia comunicativa intercultural. Así, el Instituto Cervantes la define como "la habilidad del aprendiente de una segunda lengua o lengua extranjera para desenvolverse adecuada y satisfactoriamente en las situaciones de comunicación intercultural que se producen con frecuencia en la sociedad actual, caracterizada por la pluriculturalidad" (Poradowska, 2016, p.2). En otras palabras, es la habilidad de comprender y relacionarse con personas que no comparten la misma cultura, etnia, lengua u otras variables. En este sentido, el Marco Común Europeo de Referencia para las Lenguas (Consejo de Europa, 2001) hace referencia a los términos de competencia plurilingüe y pluricultural para referirse a la capacidad de utilizar las lenguas con fines comunicativos y de participar en una relación intercultural, en la que una persona posee experiencia de diferentes lenguas y sus culturas. Según Byram (2008), la competencia comunicativa intercultural incluye una serie de actitudes, conocimientos, habilidades de interpretación y comprensión, de descubrimiento e interacción y conciencia crítica cultural.

Valls (2011) especifica que la competencia comunicativa intercultural pone en funcionamiento una serie de habilidades que permiten relacionar la propia cultura con la de la lengua meta, superar estereotipos, relacionarse con personas de otras culturas y ejercer de intermediario. De esta manera, la enseñanza de una lengua extranjera pretende que los estudiantes se desarrollen como hablantes interculturales, los cuales interrelacionan todas sus capacidades lingüísticas y culturales con la intención de comprender otras culturas, de explicar la suya y de relacionarse satisfactoriamente con personas con otras culturas. Asimismo, Byram (2008) relaciona el término de "hablante intercultural" con "mediador intercultural", poniendo especial énfasis en la acción de mediar entre uno mismo y otras personas con lenguas y culturas diferentes. Para ello, se han de promover entre los alumnos actitudes de empatía, curiosidad, apertura, relativización de la propia cultura y disposición favorable al contacto con otras culturas.

Por lo tanto, uno de los objetivos fundamentales del enfoque intercultural en la enseñanza de lenguas consiste en desarrollar la personalidad del alumno al mismo tiempo que su sentimiento de identidad, gracias a la enriquecedora experiencia que supone enfrentarse a lo diferente en los ámbitos de la lengua y de la cultura (Byram, 2014; Consejo de Europa, 2001; Da Silva, 2011; Paricio-Tato, 2011). Según Byram y Fleming (2001), este enfoque intercultural se dirige no solo al país objeto de estudio, sino también al país del estudiante, facilitando el establecimiento de relaciones entre ambas. Al comparar aspectos de las dos culturas, se produce entre ellas un mayor acercamiento y entendimiento y también se desarrolla una actitud crítica en relación con la propia cultura. De este modo, para desarrollar la competencia comunicativa intercultural se debe incrementar la cantidad y calidad de los contactos entre estudiantes de diversos países.

Actualmente, debido al auge de las TIC podemos conseguir contactos que nunca antes habíamos imaginado y conectar varias clases entre sí gracias a la movilidad virtual. Esta está adquiriendo mucha importancia tanto en los centros escolares como en la Educación Superior, ya que favorece los contactos y hermanamientos entre centros permitiendo no solo mejorar la expresión oral y 
escrita, sino también aprender de ellos y con ellos (Lewis, O' Rourke y Dooly, 2016; Selçuk Akdemir, 2017).

\section{La movilidad virtual en los centros educativos}

El mundo actual está caracterizado por las relaciones internacionales y el aumento de la movilidad. Los niños en su entorno más inmediato también están en contacto con personas de diferentes culturas y orígenes, por ejemplo, en su misma aula. De ahí radica la importancia de incluir el desarrollo de la competencia comunicativa intercultural en la enseñanza de idiomas (Byram, 2008).

Según Council of Europe (2015), la movilidad consiste en el movimiento de un agente o grupo social a un espacio diferente. La escuela, como institución social, tiene la tarea de promover la movilidad individual y contribuir al cambio social. Gracias al avance de las TIC, la movilidad virtual ofrece cada vez más nuevas oportunidades a la escuela de poner en contacto a diferentes alumnos, separados por una gran distancia física, utilizando una lengua extranjera como intermediaria. El uso flexible de las redes sociales aumenta y mejora la relación entre la escuela y diferentes realidades socioculturales. Habitualmente, esta movilidad virtual adquiere la forma de proyectos interdisciplinares entre colegios de países distintos a través de diversos programas en línea. De los programas que actualmente se están llevando a cabo, los tres más importantes en el ámbito educativo son: el Programa Comenius, Connecting Classrooms y eTwinning.

El programa eTwinning se define como una "iniciativa de la Comisión Europea encaminada a promover el establecimiento de hermanamientos escolares y el desarrollo de proyectos de colaboración a través de Internet entre dos o más centros escolares de países europeos diferentes sobre cualquier tema del ámbito escolar" (INTEF, 2013-2014). De esta manera, como bien resalta Moreno (2008), eTwinning permite que varios maestros cuyos centros distan miles de kilómetros puedan acordar y realizar un proyecto de trabajo común a través de las TIC. En otras palabras, eTwinning es una ventana al mundo, a Europa, que une a las personas de la comunidad educativa porque aprenden colaborativamente favoreciendo el intercambio cultural donde todo se comparte a través de las TIC. Los tres pilares en los que se asientan los proyectos eTwinning son: la dimensión europea, el uso de las TIC y el trabajo en colaboración. Dicho de otra manera, su funcionamiento se basa en que dos o más centros escolares de diferentes países de Europa contacten para realizar un proyecto común a través de una plataforma colaborativa llamada TwinSpace, desarrollando al mismo tiempo las competencias digitales, lingüísticas y culturales.

Este portal de la Comisión Europea comenzó en el año 2005 como una parte fundamental del programa eLearning y desde 2007 está integrado en el Programa de Aprendizaje Permanente, siendo financiado actualmente por la Comisión Europea dentro del marco del programa Erasmus + . Como recogen Kearney y Gras Velázquez (2015), hay 330.000 personas registradas en la plataforma eTwinning pertenecientes a 33 países diferentes, aunque adicionalmente cubre otros países denominados eTwinningPlus.

Los proyectos eTwinning se pueden realizar con alumnos de cualquier nivel educativo, sin embargo, la mayoría se llevan a cabo en los niveles de Educación Secundaria y, en menor grado, en Primaria (Nieto Moreno de Diezmas y Ortiz Calero, 2017). En cuanto a la etapa de Infantil, encontramos en García Ruano (2013) un estudio sobre el proyecto eTwinning "Un mar de culturas" desarrollado en Infantil que evidencia que la participación de los alumnos de Infantil en estos proyectos de hermanamiento escolar genera un alto grado de motivación en los alumnos, así como el hecho de que favorece la adquisición de unos valores de respeto hacia otras culturas. Nieto Moreno de Diezmas y Ortiz Calero (2017) reflejan, además, que el impacto de un proyecto éTwinning sobre el otoño en niños de 3 a 5 años de un programa bilingüe recae principalmente en la mejora de la competencia lingüística en lengua inglesa desde una marcada perspectiva intercultural. El trabajo colaborativo entre los docentes de proyectos eTwinning en la etapa de Infantil les ayuda a abrir sus 
clases a otras culturas y a mejorar su autoestima y la conciencia de que las tareas realizadas para los niños de Infantil son compartidas con los docentes de otros países (Szulc Kurpaska, 2009).

Después de analizar el marco teórico donde se pone de manifiesto la estrecha relación entre la educación intercultural en el aprendizaje de idiomas y la movilidad virtual, nos planteamos una investigación que trate de responder a las siguientes preguntas: ¿es posible introducir la internacionalización en la Educación Infantil?, ¿qué impacto tiene la participación en un proyecto eTwinning sobre la motivación del alumnado de Infantil y de los docentes de ambos países?

\section{Desarrollo del proyecto $\boldsymbol{e} T$ winning en Educación Infantil: Let's get dressed!}

El proyecto eTwinning: Let's get dressed nace del interés de que los niños de Educación Infantil puedan conocer otras culturas sin tener que salir de su propia aula, a la vez que aprenden una lengua extranjera. Dichos valores quedan recogidos en el Decreto 122/2007, de 27 de diciembre, por el que se establece el currículo del segundo ciclo de la Educación Infantil en la Comunidad de Castilla y León. El área de Conocimiento del entorno indica que se debe fomentar el establecimiento de relaciones tolerantes, respetuosas y afectivas con niños de otras culturas. En este mismo sentido en el área de Lenguajes: comunicación y representación propone a la lengua extranjera como un vehículo de acercamiento entre culturas.

Este proyecto eTwinning: Let's get dressed se ha llevado a cabo entre dos colegios de España y Francia. En España, el centro donde tuvo lugar dicha experiencia es el CEIP Fernando de Rojas de Burgos. El aula en el que se ha realizado el proyecto es el aula de $3^{\circ}$ de Educación Infantil A. Cuentan con dos sesiones semanales de inglés, de 1 hora y 50 minutos respectivamente, impartidas por una maestra especialista de inglés. El centro educativo francés con el que se ha realizado el hermanamiento, es el colegio público École de Seux en Saint Étienne Les Remiremont. Su maestratutora es la encargada de enseñarles inglés al igual que el resto de asignaturas, dedicándole el tiempo que considere necesario a esta materia.

El proyecto Let's get dressed! tiene una duración aproximada de dos meses, desde el 19 de marzo de 2018, fecha en la que fue aprobado por los Servicios Nacionales de España y Francia y en la que se generó el TwinSpace, hasta el 19 de mayo de 2018 finalizando con la llegada de los productos finales realizados por los propios alumnos. Durante estos dos meses, se compartieron a través de la plataforma TwinSpace numerosas fotografías, vídeos, canciones, descripciones de juegos y actividades de las sesiones de inglés. El proyecto se encuentra entroncado en las áreas del Conocimiento del entorno y Lenguajes: comunicación y representación, en concreto en la enseñanza-aprendizaje de la primera lengua extranjera (inglés).

El tema del proyecto Let's get dressed! es la ropa, por ser un tema cercano y debido a que pertenece a las rutinas diarias. Asimismo, se pretende fomentar la autonomía personal de los niños en hábitos como elegir su ropa o vestirse solos. Los objetivos principales del proyecto son que los alumnos se relacionen satisfactoriamente con niños de otro país y que valoren la lengua inglesa como un medio de relación, además de aprender vocabulario, canciones y juegos sobre la ropa y las estaciones.

De entre las siete competencias clave recogidas en la Ley Orgánica de Mejora de la Calidad Educativa (LOMCE, 2013), en este proyecto se desarrollan en los alumnos, principalmente, cuatro competencias: comunicación lingüística, aprender a aprender, competencias sociales y cívicas y conciencia y expresiones culturales.

a) Comunicación lingüística: al mostrar interés por interactuar con los demás y utilizar la lengua, en este caso la lengua extranjera, como un instrumento fundamental para la socialización. Durante el proyecto, los alumnos utilizan el inglés para participar en los 
diversos juegos y actividades, a pesar de que siguen haciendo uso de su lengua materna debido a su edad.

b) Aprender a aprender: por medio de la plataforma TwinSpace los alumnos pueden aprender de otros niños de su misma edad de otro país y viceversa, y pueden enseñarles lo que ellos saben, consiguiendo así un aprendizaje cada vez más autónomo y manteniendo alta su motivación. Los niños podrán descubrir que pueden utilizar los conocimientos adquiridos en otros contextos.

c) Competencias sociales y cívicas: se fomentan interacciones entre alumnos de los dos países desarrollando actitudes de respeto y tolerancia hacia las diferencias personales y sociales de los otros.

d) Conciencia y expresiones culturales: el establecer contacto con un centro de Francia conlleva respetar su diversidad cultural y mostrar interés y aprecio por su cultura, fomentando el diálogo intercultural.

Además de estas cuatro competencias, los docentes implicados desarrollan el ámbito de la competencia digital, puesto que se fomenta el aprendizaje y la comunicación a través del uso de las TIC gracias a las posibilidades que ofrece eTwinning, valorando sus fortalezas y debilidades en todo momento y respetando los principios éticos de nuestra profesión en su uso. A continuación, se resume el cronograma de las actividades junto con la evidencia del uso del TwinSpace llevado a cabo en el proyecto.

Tabla 1

Desarrollo del proyecto eTwinning

\begin{tabular}{|c|c|c|}
\hline Actividades & Objetivos & TwinSpace \\
\hline $\begin{array}{l}\text { Actividad } 0: \\
\text { Welcome friends!! }\end{array}$ & $\begin{array}{l}\text { Conocer las expectativas de los } \\
\text { alumnos ante la posibilidad de } \\
\text { realizar un proyecto eTwinning. }\end{array}$ & $\begin{array}{l}\text { Una vez aprobado el proyecto } \\
\text { eTwinning, se publica una entrada de } \\
\text { bienvenida en el TwinSpace con el título } \\
\text { de Welcome to everybody. En ella se } \\
\text { recogen todas las expectativas que los } \\
\text { alumnos comentaron en clase al igual } \\
\text { que las del docente. Se comparte el } \\
\text { enlace de la página web del CEIP } \\
\text { Fernando de Rojas. }\end{array}$ \\
\hline $\begin{array}{l}\text { Actividad } \\
\text { introductoria: } \\
\text { Our routines in the } \\
\text { English lessons }\end{array}$ & $\begin{array}{l}\text { Compartir las rutinas de la clase } \\
\text { de inglés con los alumnos } \\
\text { franceses. }\end{array}$ & $\begin{array}{l}\text { En la entrada Spanish routines se } \\
\text { comparte una explicación de las } \\
\text { diferentes rutinas que se realizan al } \\
\text { inicio de las sesiones de inglés junto } \\
\text { con las canciones y los vídeos } \\
\text { empleados, al igual que una fotografía } \\
\text { del panel Daily routines. }\end{array}$ \\
\hline $\begin{array}{l}\text { Primera sesión: } \\
\text { It's time for new words!! }\end{array}$ & $\begin{array}{l}\text { Introducir el nuevo vocabulario } \\
\text { de la ropa. } \\
\text { Aprender la canción Clothes song. } \\
\text { Desarrollar la memoria. }\end{array}$ & $\begin{array}{l}\text { Se publica una entrada bajo el título de } \\
\text { Clothes vocabulary and song, en la que se } \\
\text { comparte una fotografía de las flashcards } \\
\text { que se utilizan en el aula y la letra y el } \\
\text { audio de la canción, para que los } \\
\text { alumnos franceses puedan aprenderla y }\end{array}$ \\
\hline
\end{tabular}




\begin{tabular}{|c|c|c|}
\hline & & bailarla. \\
\hline $\begin{array}{l}\text { Segunda sesión: } \\
\text { Let's meet our Spanish } \\
\text { friends }\end{array}$ & $\begin{array}{l}\text { Dar a conocer el proyecto } \\
\text { eTwinning en clase. } \\
\text { Aumentar la motivación de los } \\
\text { alumnos. } \\
\text { Repasar las estructuras simples } I \\
\text { am... y My name is... }\end{array}$ & $\begin{array}{l}\text { Se edita el vídeo en dos partes, una } \\
\text { primera parte con fotografías e } \\
\text { información del colegio y una segunda } \\
\text { parte con la presentación de los } \\
\text { alumnos. Posteriormente se comparte } \\
\text { en el TwinSpace bajo la entrada titulada } \\
\text { Let's meet our Spanish friends. }\end{array}$ \\
\hline $\begin{array}{l}\text { Segunda sesión: } \\
\text { Feeling bag }\end{array}$ & $\begin{array}{l}\text { Repasar el vocabulario de la } \\
\text { ropa. } \\
\text { Asociar cada palabra con su } \\
\text { grafía. } \\
\text { Fomentar el desarrollo de los } \\
\text { sentidos, en especial el tacto. } \\
\text { Ofrecer un juego de la temática } \\
\text { de la ropa a los alumnos } \\
\text { franceses. }\end{array}$ & $\begin{array}{l}\text { En la entrada Clothes games se comparte } \\
\text { una explicación de esta actividad junto } \\
\text { con fotografías de los materiales } \\
\text { empleados y de los niños realizando la } \\
\text { actividad. }\end{array}$ \\
\hline $\begin{array}{l}\text { Tercera sesión: } \\
\text { Washing line game }\end{array}$ & $\begin{array}{l}\text { Interiorizar el vocabulario de la } \\
\text { ropa. } \\
\text { Responder a la orden take ... and } \\
\text { hang... (e.g. the trousers) from the } \\
\text { washing line. } \\
\text { Desarrollar la motricidad fina de } \\
\text { los alumnos. } \\
\text { Ofrecer un juego de la temática } \\
\text { de la ropa a los alumnos } \\
\text { franceses. }\end{array}$ & $\begin{array}{l}\text { En la entrada Clothes games se comparte } \\
\text { una explicación de este juego junto con } \\
\text { fotografías y un vídeo de su realización } \\
\text { grabado en el aula. }\end{array}$ \\
\hline $\begin{array}{l}\text { Tercera sesión: } \\
\text { Let's dress the doll! }\end{array}$ & $\begin{array}{l}\text { Aprender el vocabulario de la } \\
\text { ropa. } \\
\text { Responder a la orden put on } \\
\text { his... (e.g. hat) } \\
\text { Fomentar la autonomía en } \\
\text { hábitos básicos. } \\
\text { Desarrollar la motricidad fina de } \\
\text { los alumnos. } \\
\text { Ofrecer un juego de la temática } \\
\text { de la ropa a los alumnos } \\
\text { franceses. }\end{array}$ & $\begin{array}{l}\text { En la entrada Clothes games se comparte } \\
\text { una explicación de este juego junto con } \\
\text { fotografías y un vídeo de su realización } \\
\text { grabado en el aula. De tal forma que se } \\
\text { publica una ficha con tres juegos } \\
\text { distintos. }\end{array}$ \\
\hline $\begin{array}{l}\text { Tercera sesión: } \\
\text { Story time }\end{array}$ & $\begin{array}{l}\text { Mostrar una actitud de escucha e } \\
\text { interés hacia un cuento. } \\
\text { Comprender la historia del } \\
\text { cuento. }\end{array}$ & $\begin{array}{l}\text { Se comparten fotografías del Big Book } \\
\text { en la sección de photos, para que los } \\
\text { alumnos franceses puedan leerlo, y una } \\
\text { imagen del storytelling. }\end{array}$ \\
\hline $\begin{array}{l}\text { Cuarta sesión: } \\
\text { Clothes race }\end{array}$ & $\begin{array}{l}\text { Repasar el vocabulario de la } \\
\text { ropa. } \\
\text { Mejorar su capacidad de } \\
\text { reacción. }\end{array}$ & $\begin{array}{l}\text { Se edita el vídeo que se comparte junto } \\
\text { con varias fotografías que muestran la } \\
\text { secuencia del juego y una explicación, } \\
\text { en la entrada Clothes race game. }\end{array}$ \\
\hline
\end{tabular}




\begin{tabular}{|c|c|c|}
\hline & $\begin{array}{l}\text { Ofrecer un juego de la temática } \\
\text { de la ropa a los alumnos } \\
\text { franceses. }\end{array}$ & \\
\hline $\begin{array}{l}\text { Cuarta sesión: } \\
\text { What season is it? }\end{array}$ & $\begin{array}{l}\text { Aprender el nombre de las } \\
\text { estaciones y asociarlas con su } \\
\text { imagen. } \\
\text { Relacionar las prendas de ropa } \\
\text { con su estación. } \\
\text { Aprender la canción Seasons song. }\end{array}$ & $\begin{array}{l}\text { Se publica una entrada bajo el título de } \\
\text { Seasons vocabulary and song, en la que se } \\
\text { comparten varias fotografías de las } \\
\text { flashcards que se utilizan en el aula y la } \\
\text { letra y el audio de la nueva canción, } \\
\text { para que los alumnos franceses puedan } \\
\text { aprenderla y bailarla. Además, se } \\
\text { vuelve a compartir la letra de la } \\
\text { canción de las estaciones de las rutinas. }\end{array}$ \\
\hline $\begin{array}{l}\text { Quinta sesión: } \\
\text { Watching our French } \\
\text { friends }\end{array}$ & $\begin{array}{l}\text { Conocer a los alumnos franceses } \\
\text { y su colegio. } \\
\text { Aumentar la motivación y el } \\
\text { interés de los alumnos por el } \\
\text { proyecto. }\end{array}$ & $\begin{array}{l}\text { Se crea una entrada con el título Spanish } \\
\text { feelings to our French friends, en la que se } \\
\text { comparten fotografías y un vídeo que } \\
\text { muestran el momento en el que los } \\
\text { alumnos españoles conocen a sus } \\
\text { compañeros franceses. }\end{array}$ \\
\hline $\begin{array}{l}\text { Quinta sesión: } \\
\text { Dress the cardboard doll }\end{array}$ & $\begin{array}{l}\text { Interiorizar el vocabulario de las } \\
\text { estaciones y las prendas de ropa. } \\
\text { Responder adecuadamente ante } \\
\text { las estructuras put on... y take } \\
\text { off... } \\
\text { Ofrecer un juego de la temática } \\
\text { de la ropa y las estaciones a los } \\
\text { alumnos franceses. }\end{array}$ & $\begin{array}{l}\text { Se comparte la entrada The seasons } \\
\text { change our clothes con una explicación del } \\
\text { juego y fotografías de los alumnos } \\
\text { vistiendo a la muñeca. }\end{array}$ \\
\hline $\begin{array}{l}\text { Sexta sesión: } \\
\text { Find the couple! }\end{array}$ & $\begin{array}{l}\text { Repasar el vocabulario de la } \\
\text { ropa. } \\
\text { Aprender a jugar al memory game. } \\
\text { Asociar cada prenda de ropa con } \\
\text { su palabra escrita. } \\
\text { Ofrecer un juego sobre la ropa a } \\
\text { los alumnos franceses. }\end{array}$ & $\begin{array}{l}\text { Se publica una entrada con el nombre } \\
\text { de Clothes memory game, en la que se } \\
\text { comparte una explicación del juego y } \\
\text { fotografías del material, para que lo } \\
\text { puedan realizar los compañeros } \\
\text { franceses. }\end{array}$ \\
\hline $\begin{array}{c}\text { Sexta sesión: } \\
\text { French clothes games }\end{array}$ & 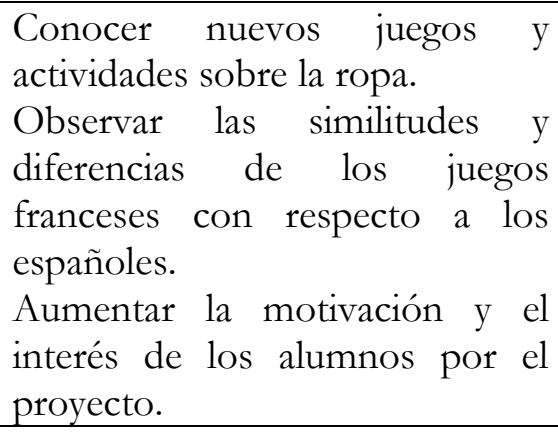 & $\begin{array}{l}\text { Se añaden fotografías en la entrada } \\
\text { Spanish feelings to our French friends de los } \\
\text { alumnos viendo los vídeos de los } \\
\text { juegos franceses. }\end{array}$ \\
\hline $\begin{array}{l}\text { Sexta sesión: } \\
\text { Singers in action }\end{array}$ & $\begin{array}{l}\text { Aprender la canción Seasons } \\
\text { song. } \\
\text { Cantar y bailar la canción siendo } \\
\text { conscientes de que va a ser vista } \\
\text { por los compañeros franceses. }\end{array}$ & $\begin{array}{l}\text { Se edita y se comparte el video de la } \\
\text { canción grabado en clase en una } \\
\text { entrada con el título de Dancing the } \\
\text { seasons song. }\end{array}$ \\
\hline
\end{tabular}




\begin{tabular}{|c|c|c|}
\hline $\begin{array}{l}\text { Séptima sesión: } \\
\text { Put on your shoes }\end{array}$ & $\begin{array}{l}\text { Escuchar y bailar la canción Put } \\
\text { on your shoes. } \\
\text { Imitar acciones de ponerse } \\
\text { diferentes prendas de ropa. }\end{array}$ & $\begin{array}{l}\text { Se comparte el vídeo de la canción de } \\
\text { Youtube en la sección de vídeos. }\end{array}$ \\
\hline $\begin{array}{l}\text { Séptima sesión: } \\
\text { Playing time with the } \\
\text { seasons pop-out }\end{array}$ & $\begin{array}{l}\text { Repasar el nombre de las } \\
\text { estaciones con pop-outs. } \\
\text { Asociar cada estación con una } \\
\text { imagen. }\end{array}$ & $\begin{array}{l}\text { Se crea la entrada Seasons pop-outs en la } \\
\text { que se comparte una imagen del pop-out } \\
\text { junto con una explicación de cómo } \\
\text { jugar y el vídeo grabado en clase. }\end{array}$ \\
\hline $\begin{array}{l}\text { Producto final: } \\
\text { Drawing for our French } \\
\text { friends }\end{array}$ & $\begin{array}{l}\text { Dibujar y diseñar su atuendo en } \\
\text { relación a su estación favorita. } \\
\text { Aumentar la motivación de los } \\
\text { alumnos con respecto al } \\
\text { proyecto eTwinning. } \\
\text { Generar emociones en los } \\
\text { alumnos españoles, al preparar } \\
\text { sus dibujos para los compañeros } \\
\text { franceses, y en los alumnos } \\
\text { franceses, al recibir cada uno un } \\
\text { dibujo de un niño español. }\end{array}$ & $\begin{array}{l}\text { Se crea una publicación Spanish drawings } \\
\text { ready to be sent para avisar de que los } \\
\text { dibujos de los niños españoles van a } \\
\text { ser enviados muy pronto y se comparte } \\
\text { una fotografía del sobre listo. A su vez, } \\
\text { la maestra francesa publica la entrada } \\
\text { Our French drawings ready to be sent!! para } \\
\text { avisar de que sus dibujos, producto } \\
\text { final, acaban de ser enviados al colegio } \\
\text { español. }\end{array}$ \\
\hline
\end{tabular}

Cabe destacar que, para la buena marcha del proyecto, ha sido necesaria una comunicación continua a través de correos personales entre las maestras colaboradoras para determinar ciertos aspectos del proyecto y establecer fechas. Sin duda alguna, este es un proyecto en el que imperan los valores de tolerancia, igualdad y respeto hacia otras culturas diferentes a la nuestra, valor muy importante en el centro español y francés, por lo que se ha pretendido fomentar en todo momento el respeto hacia otras culturas y la curiosidad por conocerlas. Los equipos directivos de ambos colegios han mantenido siempre una buena disposición y confianza en este proyecto, estando informados en todo momento y animando al resto del profesorado a participar en proyectos eTwinning en próximos cursos.

\section{Metodología}

\section{Objetivos}

Los objetivos que perseguimos en la investigación son los siguientes:

a) Conocer las percepciones de los docentes de los centros educativos sobre el desarrollo del proyecto eTwinning: Let's get dressed.

b) Identificar la valoración de los niños sobre su participación en el proyecto e Twinning.

\section{Participantes}

Los datos analizados en el presente artículo fueron recopilados de las respuestas de 47 personas, de las cuales 2 son maestras y 45 estudiantes. Entre ellos se encuentran:
a) La maestra especialista de inglés de la clase de $3^{\circ}$ Educación Infantil A del CEIP Fernando de Rojas de Burgos (España).
b) La tutora de la clase CP y CE1 de École de Seux de Saint Etienne Les Remiroment (Francia).
c) 24 alumnos españoles de entre 5 y 6 años de edad.
d) 21 alumnos franceses de entre 6 y 8 años de edad. 


\section{Procedimiento}

En la primera fase, se trata de recoger los datos de las percepciones de las profesoras sobre su participación en el proyecto. Para ello, se diseñó un cuestionario para conocer las percepciones de los docentes acerca de esta experiencia de hermanamiento eTwinning y medir puntos fuertes y débiles del proyecto. La primera parte del cuestionario está compuesta por 9 ítems que se valoran según una escala Likert de cinco puntos (1, totalmente de acuerdo; 5, totalmente desacuerdo) para facilitar los contrastes y las comparaciones. Se trata de identificar si el proyecto éTwinning Let's get dressed! está contextualizado en el currículum y en el área de lengua extranjera, si está adaptado a la edad y nivel de los alumnos, si permite desarrollar varias competencias en las diversas áreas curriculares, si las actividades son originales y atractivas para los alumnos, si los dos centros europeos implicados se han coordinado y han compartido frecuentemente materiales en el TwinSpace y si consideran que el proyecto ha supuesto un impacto positivo en los alumnos. La segunda parte consiste en cinco preguntas abiertas para conocer sus experiencias previas en participación en proyectos eTwinning, sus sensaciones en esta experiencia internacional, lo que han aprendido y cuáles han sido tanto los puntos fuertes como los puntos a mejorar en el proyecto. Este cuestionario se distribuyó al final del proyecto a ambas profesoras.

En la segunda fase, el objetivo era conocer las valoraciones de los alumnos de Infantil, tanto españoles como franceses. Se diseñó un sencillo cuestionario con cinco preguntas, cuatro cerradas y una abierta. Cada profesor distribuyó el cuestionario en la sesión final del proyecto. Cabe destacar que los cuestionarios de los niños de cada país se realizaron en sus lenguas maternas. Los alumnos de Educación Infantil completaron los cuestionarios de forma oral, siendo la maestra especialista de inglés la que preguntó de manera individual a cada uno de los alumnos para poder recoger y anotar sus respuestas. El cuestionario se compuso de las siguientes preguntas: ¿te ha gustado conocer a los niños del colegio del otro país?, ¿te ha gustado ver sus vídeos y fotos?, ¿qué juego te ha gustado más? Para facilitar la respuesta de esta pregunta, se proyectaron las fotos de ellos participando de los juegos que habían realizado durante el desarrollo del proyecto. Y, por último, ¿te gustaría conocer niños de otros países? En esta última pregunta, se les deja la posibilidad de la pregunta abierta sobre qué países les gustaría conocer a más niños.

\section{Análisis de los resultados}

Las docentes afirman que el proyecto eTwinning está contextualizado en el currículum propio de cada país y, en especial, en el área de Lengua extranjera (inglés). Además, consideran que ha sido propio para las edades de los niños ( 5 a 6 años, los españoles; y de 6 a 8 años, los franceses). Es interesante que ambas docentes están de acuerdo en que el proyecto e Twinning tiene un carácter integrador de las competencias que se han de desarrollar desde las distintas áreas de los niveles educativos de los niños. En cuanto a la originalidad de las actividades que forman parte del proyecto, los docentes las valoran en alto grado. Ambas consideran que el proyecto ha tenido un impacto muy positivo en los niños de su aula, por lo tanto, se percibe que la valoración por parte del profesorado ha mostrado una gran satisfacción tanto con el proyecto como con el desarrollo del eTwinning.

En cuanto a las preguntas abiertas, las docentes reconocen que es la primera vez que participan en este tipo de hermanamientos escolares. Ambas profesoras no habían disfrutado anteriormente de ninguna experiencia similar al proyecto eTwinning sin embargo, a partir de ahora se animarán a participar en otros proyectos ya que de esta forma han descubierto cómo poder internacionalizar la labor desarrollada en el aula y a compartir diferentes experiencias didácticas y materiales con docentes de otros países. 
La positiva valoración del profesorado se apoyó en que veían como puntos fuertes del proyecto: poner en contacto a los estudiantes con niños de otros países y hacerles ver lo importante y práctico que es aprender una lengua extranjera desde un enfoque intercultural. Además, las docentes aluden que se ha comprobado que la motivación e interés de los niños aumentaba a medida que se les introducía en el proyecto eTwinning, ya que cada vez que había noticias nuevas, ambas clases estaban impacientes por ver lo compartido en el TwinSpace.

Las dificultades que señalan son que, en ocasiones, la carga lectiva dificulta la puesta en práctica de este tipo de proyectos, porque no se dispone de horas suficientes dentro del horario lectivo para su realización. Además, la docente francesa acepta haber tenido dificultades en el manejo de las TIC. El principal punto a mejorar es el grado de implicación por ambos centros, ya que en ocasiones una de las dos partes aporta e interviene más tiempo para llevar a cabo el proyecto. Por ello, es fundamental una correcta organización y división equitativa del trabajo.

En cuanto a las valoraciones de los niños sobre su participación en el proyecto, todos, tanto españoles como franceses, han respondido positivamente a las preguntas, comprobando así el alto grado de satisfacción que ha tenido este proyecto eTwinning por su parte. Les ha gustado mucho conocer a otros niños de otro país europeo y compartir con ellos las sesiones de inglés. También sienten interés por verse ellos mismos en los diferentes vídeos e imágenes subidos al TwinSpace. Por lo tanto, el impacto en los alumnos españoles y franceses ha sido muy positivo, siendo el aumento de su motivación uno de los principales efectos de este proyecto eTwinning. Según Wastiau, Crawley y Gilleran (2011), los proyectos eTwinning implican mayor motivación, un sentido más claro de la responsabilidad, más solidaridad y un aprendizaje más eficaz.

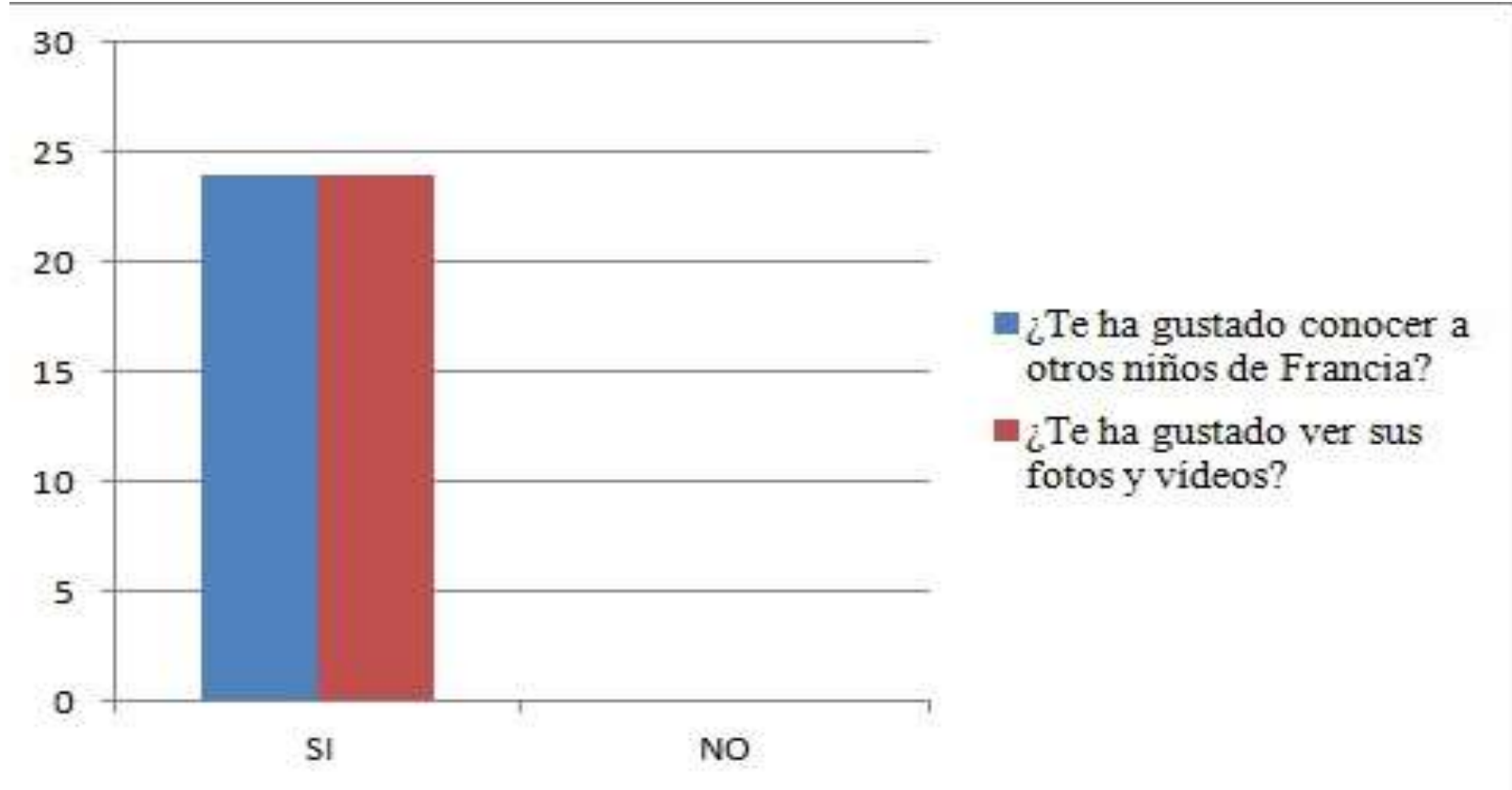

Gráfica 1. Respuestas sobre el grado de satisfacción de los alumnos españoles.

Los juegos que más han gustado a los niños españoles son: la actividad Let's dress the doll y la actividad Find the couple!; por el contrario, para los franceses es la actividad W ashing line game. Han sido los más atractivos para ellos debido a que en estos juegos los alumnos tenían un papel activo, siendo ellos los protagonistas, y a que han utilizado materiales reales, como un muñeco y su ropa. En cambio, el juego menos popular ha sido la actividad Dress the cardboard doll, pues ningún niño lo ha elegido como su favorito. 


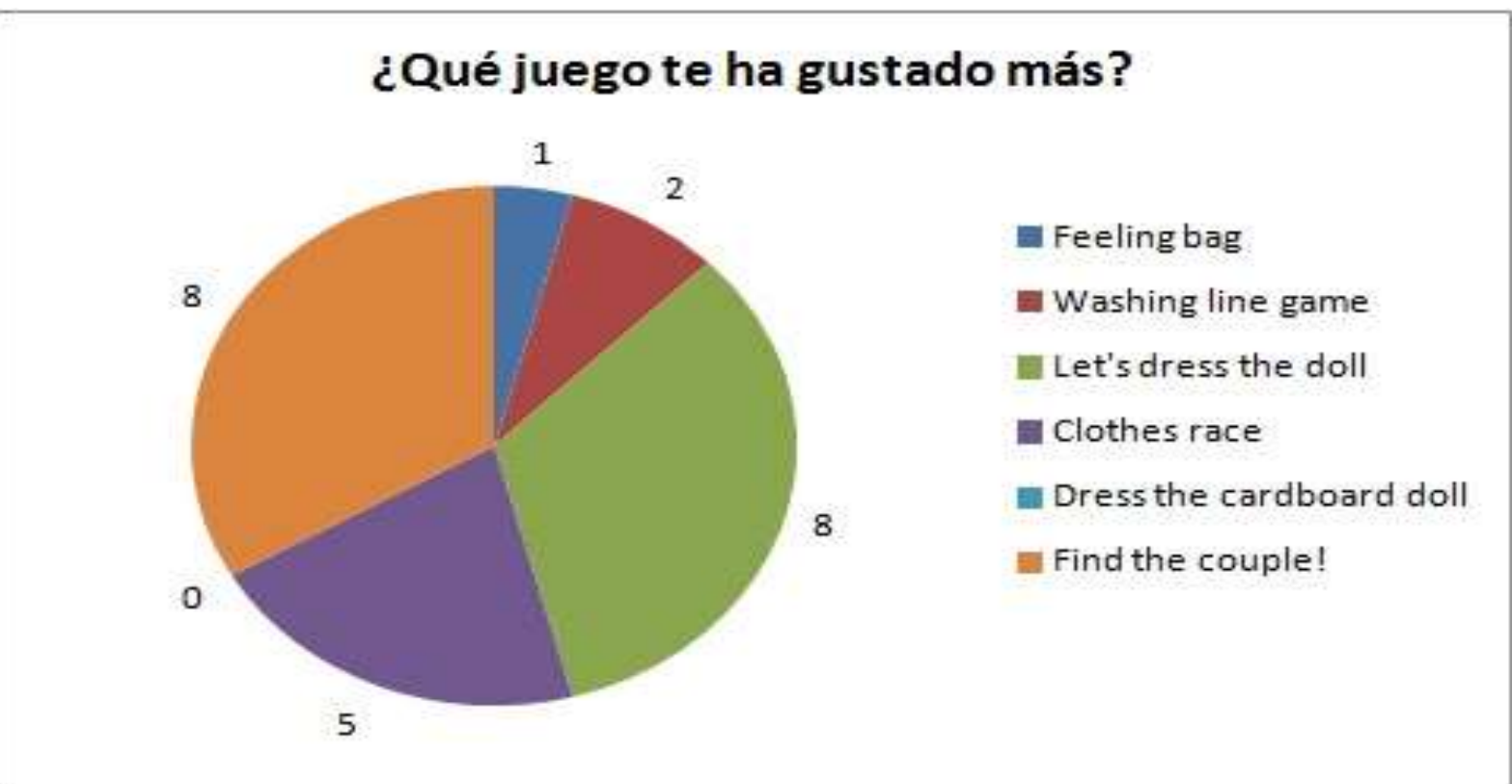

Gráfica 2. Juegos más populares entre los alumnos españoles.

Desde este momento, los alumnos españoles quieren seguir conociendo a niños de otros países, entre los que cinco alumnos volvieron a mencionar Francia y, en concreto, París. Otros países que escogieron fueron Inglaterra, Rumania, Senegal, Argentina e Italia, entre otros. Apenas coincidieron en sus respuestas. Por el contrario, en la clase francesa no tienen ninguna preferencia de países, aunque les encantaría volver a repetir con España porque algunos de sus alumnos han estado en nuestro país de vacaciones o tienen familiares viviendo aquí. Dijeron, tal y como recogió la maestra francesa "nos gustaría visitarles algún día".

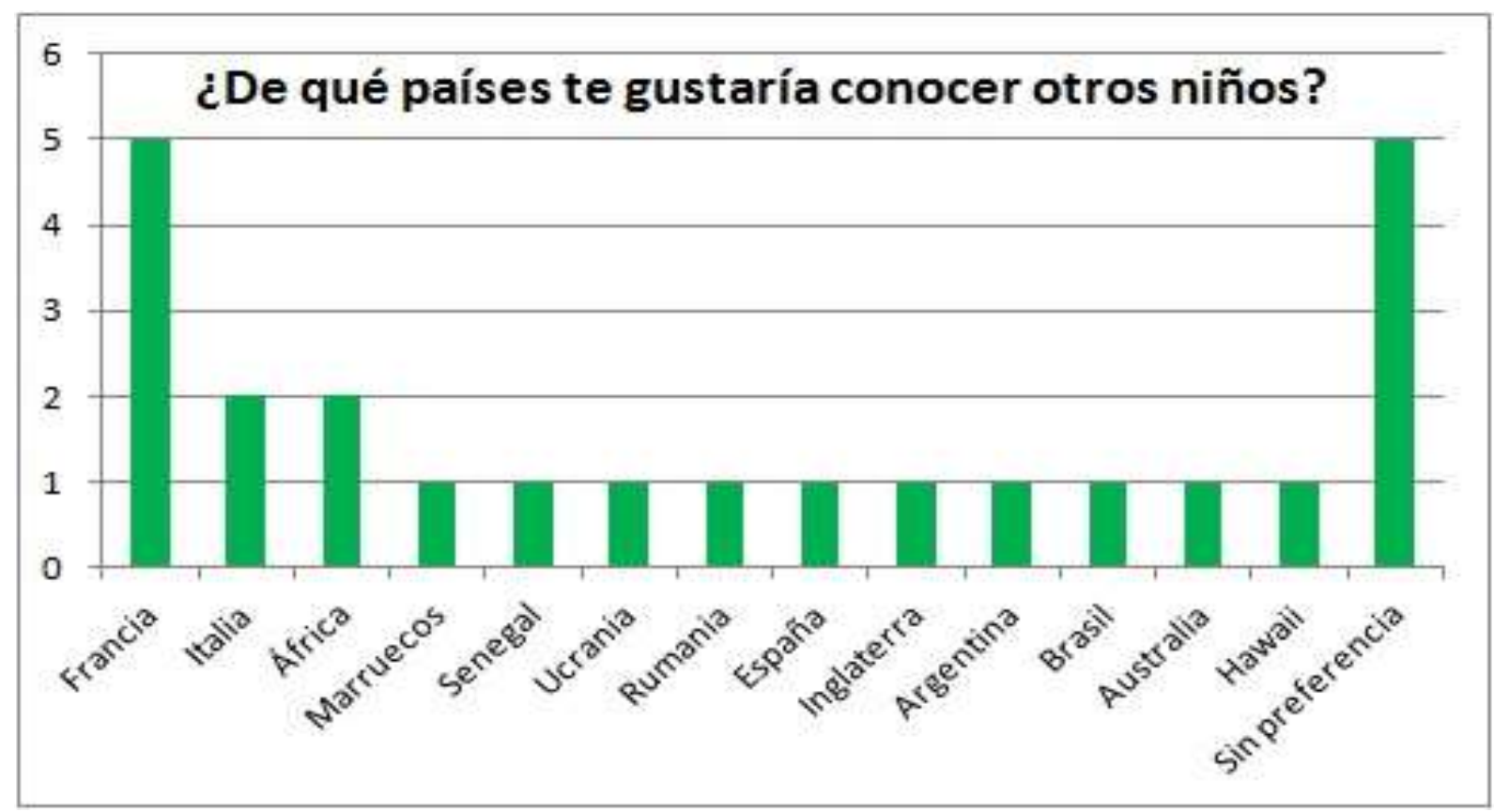

Gráfica 3. Preferencias de los alumnos españoles por conocer a otros niños. 


\section{Discusión y conclusiones}

Finalmente, se ha de resaltar que tal y como nos planteábamos en la primera pregunta de investigación, sí ha sido posible incorporar la internacionalización en la etapa de Educación Infantil a través del desarrollo del proyecto eTwinning Let's get dressed!, realizado entre el colegio Fernando de Rojas de Burgos (España) y en el colegio École de Seux de Saint Etienne Les Remiroment (Francia). Su puesta en práctica se ha realizado de forma realmente satisfactoria para todos: alumnos, docentes y centros implicados de estos dos países europeos.

Tratando de responder a la segunda pregunta de investigación, ha sido una experiencia altamente gratificante que ha aportado beneficios significativos a todos sus participantes. Esta experiencia internacional ha tenido un gran valor educativo en el aula de lengua extranjera en Educación Infantil.

Los alumnos han descubierto la necesidad de aprender inglés para poder comunicarse con otras personas con culturas diferentes, viendo de este modo la utilidad práctica del aprendizaje de las lenguas extranjeras. De este modo son capaces, a esta temprana edad, de valorar la lengua inglesa como un medio de relación con niños de otro país europeo. Estos resultados coinciden con los de Nieto Moreno de Diezmas y Ortiz Calero (2017) y Szulc-Kurpaska (2009), quienes afirman que la participación de los niños en Infantil favorece la mejora de la competencia lingüística en la lengua extranjera desde un enfoque intercultural, ya que permite a los niños de estas edades acercarse a realidades diferentes a las suyas.

Subrayamos el alto grado de motivación que muestran los niños por participar en el proyecto y que, claramente, se extiende a una actitud positiva hacia el inglés como lengua extranjera, ya que se ha podido observar incluso el cambio de actitud de algunos niños en las sesiones de inglés debido al interés suscitado gracias al proyecto eTwinning, tal y como se refiere García Ruano (2013) en su trabajo sobre el fomento del plurilingüismo a través de un proyecto eTwinning en Infantil.

Para los docentes implicados este proyecto éTwinning, ha tenido un gran valor educativo, puesto que no habían participado previamente en ninguna experiencia similar. Juntos, y de manera autodidáctica, han aprendido a manejar este portal de la Comisión Europea, lo que les ha permitido mejorar en su competencia digital, aunque uno de ellos mencionó como debilidad la falta de competencia digital. Selçuk Akdemir (2017) identifica esta debilidad como uno de los problemas más frecuentes a los que se enfrentan los docentes a la hora de llevar a cabo un proyecto eTwinning. Han descubierto las posibilidades que ofrece eTwinning, destacando no sólo los beneficios que conlleva en el aula con sus alumnos, sino también a nivel personal. Les ha puesto ante sus ojos un grupo de profesores y compañeros de diversas nacionalidades y culturas, pero con los mismos intereses y con los que se pueden realizar proyectos eTwinning en un futuro cercano (Szulc-Kurpaska, 2009).

Finalmente, para los dos centros educativos ha supuesto una experiencia totalmente innovadora, ya que anteriormente no se había participado en un proyecto eTwinning en ninguno de los dos centros. Ambos colegios esperan seguir enriqueciéndose de este tipo de experiencias internacionales en Infantil en posteriores proyectos. Este proyecto puede ser replicable en otros contextos, es decir, se puede poner en práctica en otras aulas de Educación Infantil porque es fácilmente adaptable y personalizable. Sin embargo, cabe destacar que sería necesario seguir investigando en el potencial que ofrece la herramienta TwinSpace en los proyectos eTwinning en Educación Infantil por las múltiples posibilidades que ofrece para favorecer la estrecha colaboración entre niños de otros países mediante grupos cooperativos internacionales, con el fin de que los niños adquieran un mayor protagonismo en el proyecto eTwinning. 


\section{Referencias}

Byram, M. (2014). Twenty-five years on-from cultural studies to intercultural citizenship. Language, culture and curriculum., 27(3), 209-225.

Byram, M. y Fleming, M. (2001). Perspectivas interculturales en el aprendizaje de idiomas. Enfoques a través del teatro y la etnografía. Madrid, España: Cambridge University Press.

Consejo de Europa (2001). Marco Común Europeo de Referencia para las Lenguas: aprendizaje, enseñanza, evaluación. Madrid: Ministerio de Educación, Cultura y Deporte. Disponible en: https://cvc.cervantes.es/ensenanza/biblioteca_ele/marco/cvc_mer.pdf

Council of Europe (2015). Education, mobility, otherness. The mediations functions of school. Council of Europe. Disponible en:

https://www.coe.int/t/dg4/Linguistic/Source/LE_texts_Source/LE\%202015/EducationMobility-Otherness_en.pdf

Da Silva, M. D. P. C. (2011). The "Sign up and Connect" e'Twinning project: an intercultural approach to teaching English as a foreign Language. International Conference the Future of Education. Florence, Italy

Decreto 122/2007, de 27 de diciembre, por el que se establece el currículo del segundo ciclo de la Educación Infantil en la Comunidad de Castilla y León. Boletín Oficial de Castilla y León, núm. 1, pp. 6-17. Recuperado de http://bocyl.jcyl.es/boletines/2008/01/02/pdf/BOCYL-D02012008-2.pdf

European Council (2017). European Council meeting (14 December 2017)-Conclusions. Disponible en: https://www.consilium.europa.eu/media/32204/14-final-conclusions-rev1-en.pdf

García-Ruano, M. (2013). Proyecto e Twinning: trabajo de equipo docente para el fomento del plurilingüismo en Educación Infantil por medio de las TIC. Edetania: estudios y propuestas socioeducativas, 44, 59-272.

INTEF, Instituto Nacional de Tecnologías Educativas y de Formación del Profesorado. (2014). Material de Formación Curso e'Twinnning 2013-2014. Disponible en: http://etwinning.cnice.mec.es/apls/cursos/curso_es/index.php/P\%C3\%A1gina_Principal

Kearney, C. y Gras-Velázquez, À. (2015). Una década de e'Twinning: repercusión en las prácticas, habilidades y oportunidades de desarrollo profesional de sus docentes, contado por ellos mismos. Bruselas: Servicio Central de Apoyo eTwinning-European Schoolnet. Disponible en: https://www.etwinning.net/eun-files/eTwinningreport_ES.pdf

Leiva, J. (2010). La educación intercultural entre el deseo y la realidad: reflexiones para la construcción de una cultura de la diversidad en la escuela inclusiva. Revista Docencia e Investigación, 20, 149-182. Disponible en: https://ruidera.uclm.es/xmlui/handle/10578/8314

Ley Orgánica 8/2013, de 9 de diciembre, para la Mejora de la Calidad Educativa (LOMCE). Boletín Oficial del Estado, núm. 295. Recuperada de https://www.boe.es/buscar/pdf/2013/BOE-A2013-12886-consolidado.pdf

Lewis, T., O' Rourke, B. \& Dooly, M. (2016). Innovation in language learning and teaching-Online Intercultural Exchange. Innovation in Language Learning and Teaching, 10:1,1-5.

Moreno, B. (2008). La acción e'Twinning: situación actual y necesidades formativas del profesorado. DIM: Didáctica, Innovación y Multimedia, 12. Disponible en: https://www.raco.cat/index.php/DIM/article/view/123630.

Nieto-Moreno de Diezmas, E. y Ortiz-Calero, C. (2017). CLIL e interculturalidad en Educación Infantil: aprendiendo por proyectos. Multiárea. Revista de Didáctica, 9, 24-37.

O'Dowd, R. (2015). The competences of the telecollaborative teacher. The Language Learning Journal, 43(2), 194-207. 
Paricio Tato, $\mathrm{M}^{\mathrm{a}}$ S. (2011). Contribución de las lenguas extranjeras al desarrollo de actitudes de tolerancia y respeto hacia el "otro". LINGVARVMARENA, 2, 79-89.

Poradowska, A. (1 de julio de 2016). Actividades para desarrollar la competencia intercultural en la clase de ELE. Taller presentado en la VIII y IX Jornadas Didácticas del Instituto Cervantes de Manchester (2015-2016). Manchester: Instituto Cervantes. Disponible en: https://cvc.cervantes.es/ensenanza/biblioteca_ele/publicaciones_centros/PDF/manchester_2 015-2016/09_poradowska.pdf

Sevilla-Pavón, A. y Haba Osca, J. (2016). Te das cuenta de que el mundo puede ser tan distinto y similar al mismo tiempo. Telecolaboración y desarrollo de la competencia intercultural en la educación superior. Didáctica. Lengua y Literatura, 28, 263-284.

Selçuk-Akdemir, A. (2017). eTwinning in Language Learning: The Perspectives of Successful Teachers. Journal of Education and Practice, 8(10), 182-190.

Szulc-Kurpaska, M. (2009). e'Twinning Projects in Preschool. e'Twinning-A way to education of the future. Foundation for the Development of the Education System: European Commission.

UNESCO (2006). Directrices de la UNESCO sobre la educación intercultural. París: UNESCO. Recuperado de http://unesdoc.unesco.org/images/0014/001478/147878s.pdf

Valls, L. (2011). Enseñanza/Aprendizaje de la competencia comunicativa intercultural y análisis de actitudes. Marco Ele Revista de Didáctica Ele, 13. Disponible en: http://marcoele.com/descargas/13/vallls-competencia.intercultural.pdf

Wastiau, P., Crawley, C. y Gilleran, A. (2011). Los alumnos en e'Twinning. Casos prácticos sobre la participación del alumnado. Bruselas: Central Support Service for e'Twinning. Disponible en: https://www.etwinning.net/files/ES_eTwinning_Report_2012.pdf

\section{Información sobre los autores}

Autor: Belén Zamanillo Mateo

Institución: Universidad de Burgos

Email: belenzamanillomateo@gmail.com

Autor: Esther Sanz de la Cal

Institución: Universidad de Burgos

Email: esanz@ubu.es

Autor: Josefa Ramos Rodríguez

Institución: CEIP Fernando de Rojas (Burgos, España)

Email: sanpepavi@yahoo.es 


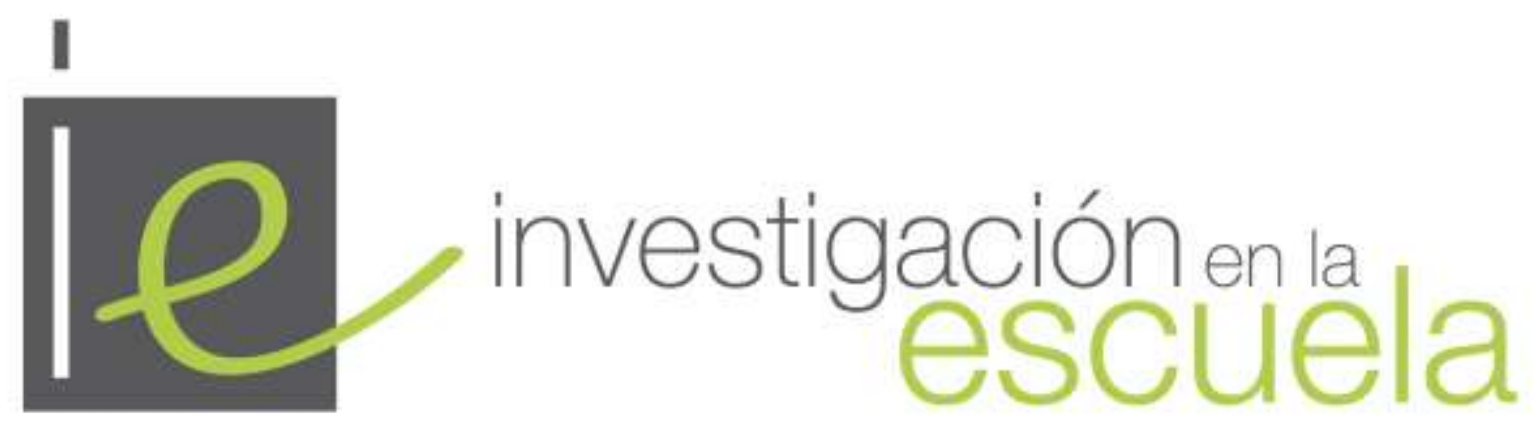

Revista académica evaluada por pares y de acceso abierto

Número 96

31 de diciembre de 2018

ISSN 2443-9991

\section{(C)}

SOMREFIIGHISRESERVEDLOS/as lectores/as pueden copiar, mostrar, y distribuir este artículo, siempre y cuando se de crédito y atribución al autor/es y a Investigación en la Escuela, se distribuya con propósitos no-comerciales, no se altere o transforme el trabajo original. Más detalles de la licencia de CreativeCommons se encuentran en http://creativecommons.org/licenses/by-nc-sa/3.0 Cualquier otro uso debe ser aprobado en conjunto por el autor/es, o Investigación en la Escuela.

ำ investigacion-en-la-escuela

Contribuya con comentarios y sugerencias en la web de la revista. Por errores y sugerencias contacte a secretaria@investigacionenlaescuela.es 


\section{Investigación en la escuela}

Consejo de dirección: Ana Rivero García (Universidad de Sevilla), Nicolás de Alba Fernández (Universidad de Sevilla), Pedro Cañal de León (Universidad de Sevilla), Francisco F. García Pérez (Universidad de Sevilla), Gabriel Travé González (Universidad de Huelva), Francisco F. Pozuelos Estrada (Universidad de Huelva)

Dirección: Ana Rivero García y Nicolás de Alba Fernández

Secretaría de edición: Elisa Navarro Medina

\section{Consejo editorial}

José Félix Angulo Rasco. Universidad de Cádiz Rosa Ma Ávila Ruiz. Universidad de Sevilla Pilar AzcárateGoded. Universidad de Cádiz Juan Bautista Martínez Rodríguez. Universidad de Granada

Nieves Blanco García. Universidad de Málaga Fernando Barragán Medero. Universidad de La Laguna José Carrillo Yáñez. Universidad de Huelva José Contreras Domingo. Universidad de Barcelona. Luis C. Contreras González. Universidad de Huelva Ana $\mathbf{M}^{\mathbf{a}}$ Criado García-Legaz. Universidad de Sevilla Rosario Cubero Pérez. Universidad de Sevilla José $\mathbf{M}^{\mathbf{a}}$ Cuenca López. Universidad de Huelva Jesús Estepa Giménez. Universidad de Huelva Rafael Feito Alonso. Universidad Complutense (Madrid)

Francisco José García Gallardo. Universidad de Huelva

Soledad García Gómez. Universidad de Sevilla J. Eduardo García Díaz. Universidad de Sevilla
Fernando Hernández Hernández. Universidad de Barcelona

Salvador Llinares Ciscar. Universidad de Alicante Alfonso Luque Lozano. Universidad de Sevilla Rosa Martín del Pozo. Universidad Complutense (Madrid)

José Martín Toscano. IES Fernando Herrera (Sevilla) Jaume Martínez Bonafé. Universidad de Valencia F. Javier Merchán Iglesias. Universidad de Sevilla Emilia Moreno Sánchez. Universidad de Huelva. Rosario Ortega Ruiz. Universidad de Córdoba Antonio de Pro Bueno. Universidad de Murcia Fco. de Paula Rodríguez Miranda. Universidad de Huelva

Pedro Sáenz-López Buñuel. Universidad de Huelva Antoni Santisteban Fernández. Universidad Autónoma (Barcelona)

Emilio Solís Ramírez. Catedrático de IES. $\mathbf{M}^{\mathbf{a}}$ Victoria Sánchez García. Universidad de Sevilla. Magdalena Suárez Ortega. Universidad de Sevilla

\section{Consejo asesor}

Manuel Área Moreira. Universidad de La Laguna Jaume Carbonell. Director Cuadernos de Pedagogía. Barcelona César Coll. Universidad de Barcelona

Christopher Day. Universidad de Nothingham. U.K.

Juan Delval. Universidad Nacional de Educación a Distancia

John Elliott. Universidad de East Anglia. Norwich. U.K.

José Gimeno Sacristán. Universidad de Valencia

André Giordan. Universidad de Paris VII y Ginebra

Francisco Imbernón. Universidad de Barcelona Ángel Pérez Gómez. Universidad de Málaga

Rafael Porlán Ariza. Universidad de Sevilla

Francesco Tonucci. Instituto de Pedagogía del C.N.R. Roma

Jurjo Torres Santomé. Universidad de A Coruña 
